\title{
Large and Local Magnetoresistance in a State-of- the-Art Perpendicular Magnetic Medium
}

This article was published in the following Dove Press journal:

Nanotechnology, Science and Applications

\author{
Morgan Williamson ${ }^{1,2}$ \\ Cheng Wang ${ }^{3}$ \\ Pin-Wei Huang ${ }^{3}$ \\ Ganping Ju ${ }^{3}$ \\ Maxim Tsoi ${ }^{1,2}$
}

'Physics Department, University of Texas at Austin, Austin, TX 787/2, USA; ${ }^{2}$ Texas Materials Institute, University of Texas at Austin, Austin, TX 787/2, USA:

${ }^{3}$ Fremont Research Center, Seagate

Technology, Fremont, CA 94538, USA
Correspondence: Maxim Tsoi

Email tsoi@physics.utexas.edu
Purpose: Magnetotransport properties of granular oxide-segregated $\mathrm{CoPtCr}$ films were studied on both macroscopic and microscopic length scales by performing bulk and pointcontact magnetoresistance measurements, respectively. Such a perpendicular magnetic medium is used in state-of-the-art hard disc drives and, when combined with magnetotransport phenomena for read/write operations, may lead to a novel concept for magnetic recording with high areal density.

Materials and Methods: The CoPtCr films were deposited by an epitaxy-like sputtering and contained several perpendicularly magnetized granular-media layers with different coercivities; they are very much like the state-of-the-art perpendicular magnetic medium, which can be found in today's hard disc drives. Magnetoresistive properties of bulk films were assessed by measuring the film resistance in the standard Van der Pauw geometry, while the local transport was probed by the point-contact technique.

Results: The bulk measurements showed only a negligible magnetoresistance of less than $0.02 \%$. In contrast, the local point-contact measurements revealed giant-magnetoresistancelike changes $\Delta R$ in local resistance of the contact $R$ with more than $10,000 \%$ ratio $\Delta R / R$.

Conclusion: The observed large and local magnetoresistive effect could be tentatively attributed to a tunnel magnetoresistance between oxide-segregated $\mathrm{CoPtCr}$ grains with different coercivities. The tunneling picture of electronic transport in our granular medium was confirmed by the observation of tunneling-like current-voltage characteristics of the contacts and bias dependence of the contact magnetoresistance - both the local point-contact resistance and magnetoresistance were found to decrease with the applied dc bias.

Keywords: magnetoresistance, granular magnetic medium, tunnel magnetoresistance, hard disc drives

\section{Introduction}

Magnetic data storage has been pushing scientific innovations and technology limits for more than half a century and has grown into a multi-billion-dollar industry today. ${ }^{1}$ The recording of information in a magnetic medium, eg a thin magnetic film in a hard disk drive (HDD), is achieved by switching its magnetization locally between two different orientations, which represent " 1 " and " 0 " of digitally stored information. The way of storing bit information in a granular medium with high magnetic anisotropy $\left(\mathrm{K}_{\mathrm{U}}\right)$ is by far the most cost-effective technology for highdensity data storage and has a clear road map of extendibility up to $2 \mathrm{~TB} / \mathrm{in}^{2}{ }^{2}$ Such granular systems with high scalability and desired thermal stability can also be potentially leveraged into fabricating memory cells of non-volatile magnetic memory such as magnetic random access memory (MRAM). We thus envision 
a merging of hard drive granular medium and the MRAM concepts into a novel magnetic memory where reading and writing operations will be achieved by magnetoresistive transport means like in MRAM, while the thermally stable perpendicularly magnetized grains with diameters of below $10 \mathrm{~nm}$ provide extraordinary scaling potential for memory applications.

In this letter, we explore magnetoresistive effects in the state-of-the-art perpendicular magnetic medium of today's HDDs - granular oxide-segregated CoPtCr films deposited on glass substrates. We use point-contact technique ${ }^{3}$ to characterize the local transport in the film composed of granular layers with different coercivities. When the relative orientation of layers changes under the influence of an externally applied magnetic field, we observe large $(>10,000 \%)$ variations in the point-contact resistance. Interestingly, the magnetoresistance is essentially absent on the macroscopic scale - bulk resistivity measurements performed on the same films showed practically no magnetoresistance $(<0.02 \%)$. The observed local and large magnetoresistance was found to decrease with the value of dc bias applied to the contact. Such variations are consistent with a tunnel magnetoresistance between individual $\mathrm{CoPtCr}$ grains and support the potential of the granular medium for future magnetic memory technology.

\section{Materials and Methods}

In our experiments we have tested a number of thin-film samples deposited by the media development team at Fremont Research Center, Seagate Technology with various layer combinations, compositions, and individual layer thicknesses ranging from 0 to $100 \AA$. In what follows we focus mainly on the magnetoresistive behavior of a particular multilayer/sample: Ru (180)/M1 (100)/Spacer (15)/M2 (100)/Spacer (15)/M3a (100)/M3b (10) where all numbers in brackets are thickness in $\AA$; this multilayer sample has a $63 \mathrm{~nm}$ thick adhesion/seed layer underneath, and a $6 \mathrm{~nm}$ thick continuous $\mathrm{CoPtX}$ capping layer ( $\mathrm{X}$ is combination of non-magnetic diluting elements such as $\mathrm{Cr}$, $\mathrm{B}$, and $\mathrm{Ru}$ ). M1, M2, M3a, and M3b are perpendicularly magnetized granular-media layers with different coercivities and a columnar structure of the metallic grain cores. The columnar structure is achieved by an epitaxy-like sputtering deposition ${ }^{4}$ of the $\mathrm{CoPtCr}_{\text {alloy }}{ }^{5}$ on top of a Rurich columnar growth template. Spacer layers are Ru-rich materials with oxide contents and are inserted to break the interlayer exchange coupling between adjacent ferromagnetic layers. Individual magnetic grains are segregated by a mixture of $\mathrm{TiO}_{2}, \mathrm{SiO}_{2}$ and other oxides. Figure $1 \mathrm{~A}$ shows a top-down planar-view transmission electron microscopy (TEM) image of the granular composite media. Figure 1B shows the magneto-optical Kerr effect (MOKE) hysteresis loop of a single magnetic layer of the granular medium. The Kerr signal confirms the perpendicular anisotropy of the material and displays the effects of the magnetic grain switching field distribution and demagnetizing field anisotropy as evidenced by the shearing of the major hysteresis loop. Figure 1C shows a result of micromagnetic modeling which simulates the hysteresis behavior of such a granular system. The modeling parameters were setup as follows: 656 Voronoi grains are generated, with the mean and coefficient of variation of grain-to-grain pitch being $7.8 \mathrm{~nm}$ and $16.7 \%$, respectively. The $0 \mathrm{~K}$ saturation magnetization and anisotropy field of the medium are $650 \mathrm{emu} / \mathrm{cc}$ and $9.2 \mathrm{kOe}$. Demagnetization and thermal effects $(300 \mathrm{~K})$ were taken into account during
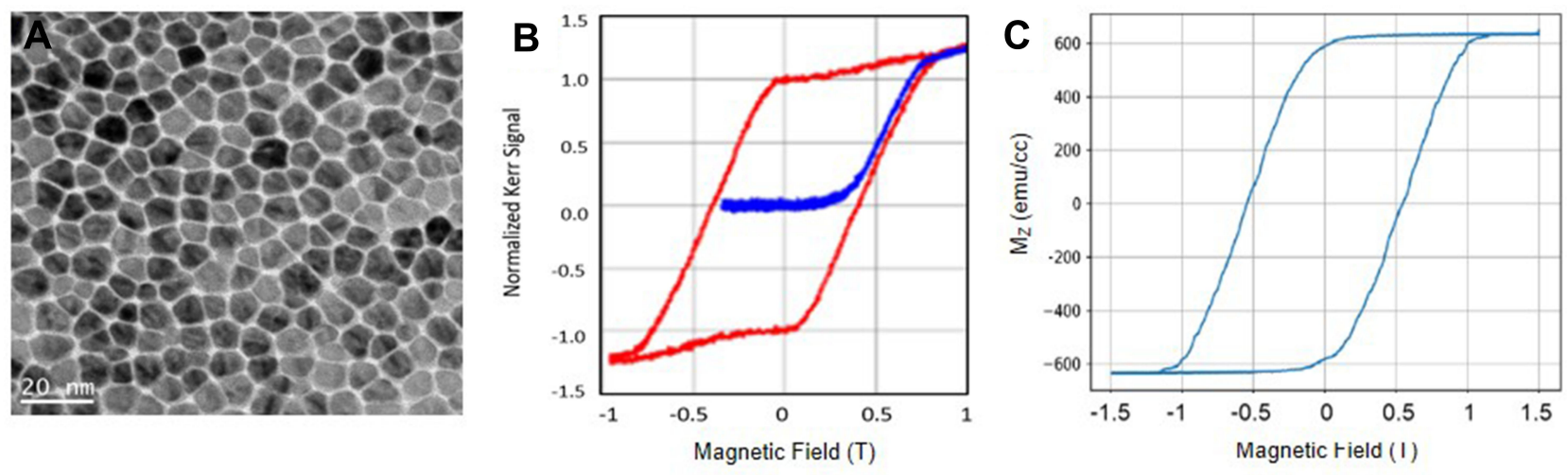

Figure I (A) Top-down bright field planar-view TEM image of the granular composite media. (B) Magnetization loop of the granular film obtained by the polar Kerr effect. Note that the external field is slightly tilted away from the normal to the film in order to highlight the thermal decay (see the slope at higher fields). (C) Micromagnetic modeling of the hysteresis (major) loop in our granular medium. 
the simulation. The damping constant is set to be 0.2 in order to reach equilibrium state within a reasonable computational time. The modeling shows that there are various portions of grains that get reversed at low, median, and high fields, indicating nucleation, coercivity, and saturation, respectively.

Magnetoresistive properties of bulk films were assessed by measuring the film resistance in the Van der Pauw geometry: four-point probes (wire-bonds) were placed in the corners of a $10 \times 10 \mathrm{~mm}^{2}$ square-shaped media film. A manual ultrasonic West-Bond wire bonder was used to make the probes; Keithley 2400 SourceMeter and Keithley 2000 Digital Multimeter were used to source current and measure voltage, respectively. An external magnetic field could be applied perpendicular to the film's plane (FPP-orientation) or in the plane of the film (FIP-orientation). Local magnetotransport properties of the same thin-film samples were assessed by point contacts used as a probe of small sample volumes $\left(10^{3}-10^{9} \mathrm{~nm}^{3}\right)$ typically associated with such contacts. The point contact was made by bringing a sharpened metallic $(\mathrm{Cu}$ or $\mathrm{W})$ tip into contact with the multilayer using a differential screw mechanism; details of the point-contact setup can be found here. ${ }^{6}$ This mechanical point-contact technique enables us to produce electrical contacts of only a few nanometers in diameter $(a)$ and probe electrical transport in very small sample volumes $\sim a^{3}$. In this work we focus on de transport measurements of point contacts in magnetic fields $B$ up to $0.7 \mathrm{~T}$ applied in the FPP-orientation and dc biases $I$ up to $1.5 \mathrm{~mA}$. All measurements were done at room temperature.

\section{Results and Discussion}

We began by investigating the bulk transport properties of our samples. All samples showed a very small magnetoresistance (MR) with MR ratio $(\Delta R / R)$ of about $0.02 \%$ in the FIP geometry where the current flows primarily along the magnetic field direction. In this paper, the MR ratio is expressed as $\Delta R / R$ where $\Delta R$ is the change in the electrical resistance due to an applied magnetic field $\left(\Delta R=R_{\max }-R_{\min }\right.$ is the difference between maximum and minimum resistance) and $\mathrm{R}$ is the minimum resistance $\left(R=R_{\min }\right)$. No MR was detected in the FPP orientation, and a continuous transition (decrease) in MR behavior was found between FIP and FPP orientations as a function of the field angle.

Contrary to the bulk measurements, point-contact measurements with $\mathrm{Cu}$ tips revealed a larger $(\sim 1 \%) \mathrm{MR}$ in both the FPP and FIP orientations. Small variations in the point-contact resistance under a varying magnetic field confirm that the magnetostrictive effect at the interface between the high anisotropy granular medium and pointcontact tip is negligible. At the same time, the relative softness of the copper tip material compared to the hard media sample can be at the origin of a relatively small MR ratio. In order to make stable electrical contact with the sample, a high amount of pressure visibly deformed sharpened $\mathrm{Cu}$ tips thus resulting in large contact areas and, consequently, involving many individual grains into the contact region. Due to the complications associated with the relatively soft copper tips, we have switched to tungsten as the point-contact tip material for better mechanical robustness.

Figure 2 shows the resistance of a tungsten point contact under a $0.7 \mathrm{~mA}$ bias as a function of an applied magnetic field in the FPP orientation. Starting down from high positive fields (red curve) the contact resistance gradually decreases from $\sim 25 \Omega$ to $\sim 20 \Omega$. The resistance of $20 \Omega$ corresponds to about $5 \mathrm{~nm}$ size of a metallic point contact in the diffusive conduction regime. ${ }^{7}$ As the applied field is decreased below $\sim 100 \mathrm{mT}$ the resistance raises to a relatively high resistance value of $\sim 30 \Omega$, demonstrating a $50 \%$ change in resistance. At negative fields, the contact resistance remains at this relatively high value down to $\sim$ $-200 \mathrm{mT}$ and then decreases back to the low value of $\sim 20 \Omega$. At high negative fields, the resistance increases again to about $25 \Omega$. The up-sweep (black curve) shows a marked

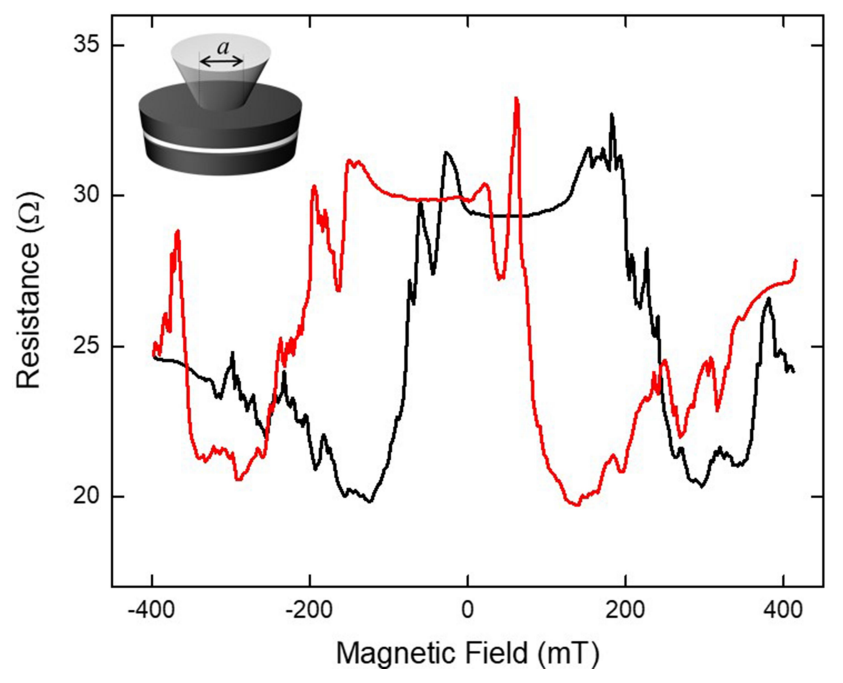

Figure 2 Point-contact magnetoresistance at $0.7 \mathrm{~mA}$ bias for a $\pm 400 \mathrm{mT}$ magneticfield sweep displaying 50\% MR ratio. Red (black) curve depicts magnetic-field downsweep (upsweep). The inset shows schematically a point contact between a sharpened $W$ tip (top light grey) and the sample with two magnetic layers (dark grey) separated by a nonmagnetic spacer (light grey). 
symmetry to the down-sweep (red curve) replicating both low $\left(R_{L}\right)$ and high $\left(R_{H}\right)$ resistance states. This behavior was observed in a dozen of tungsten point contacts with different resistances and is similar to the switching between the parallel $\left(R_{L}\right)$ and antiparallel $\left(R_{H}\right)$ states in a GMR-like storage element, eg, spin valve. The hysteretic MR behavior is symmetric about the zero applied field but with a properly tuned exchange bias field of $\sim 150 \mathrm{mT}$ could potentially be utilized in a field-toggled device with $50 \%$ MR ratio.

Along with the major reproducible features, like the high and low resistance states, MR traces show a fine structure that may vary from sweep to sweep. We illustrate the reproducibility and variability of MR traces in Figure 3 which shows major (black/gray) and minor (colored) MR loops for a tungsten point contact under a $0.7 \mathrm{~mA}$ bias. Solid (dotted/dashed) curves depict magnetic field downsweeps (up-sweeps). Each measurement starts at a positive field (just over $+400 \mathrm{mT}$ ); the field is swept down to a turning-point field (between -400 and $100 \mathrm{mT}$ ) and then back up. Minor loops with different turning points

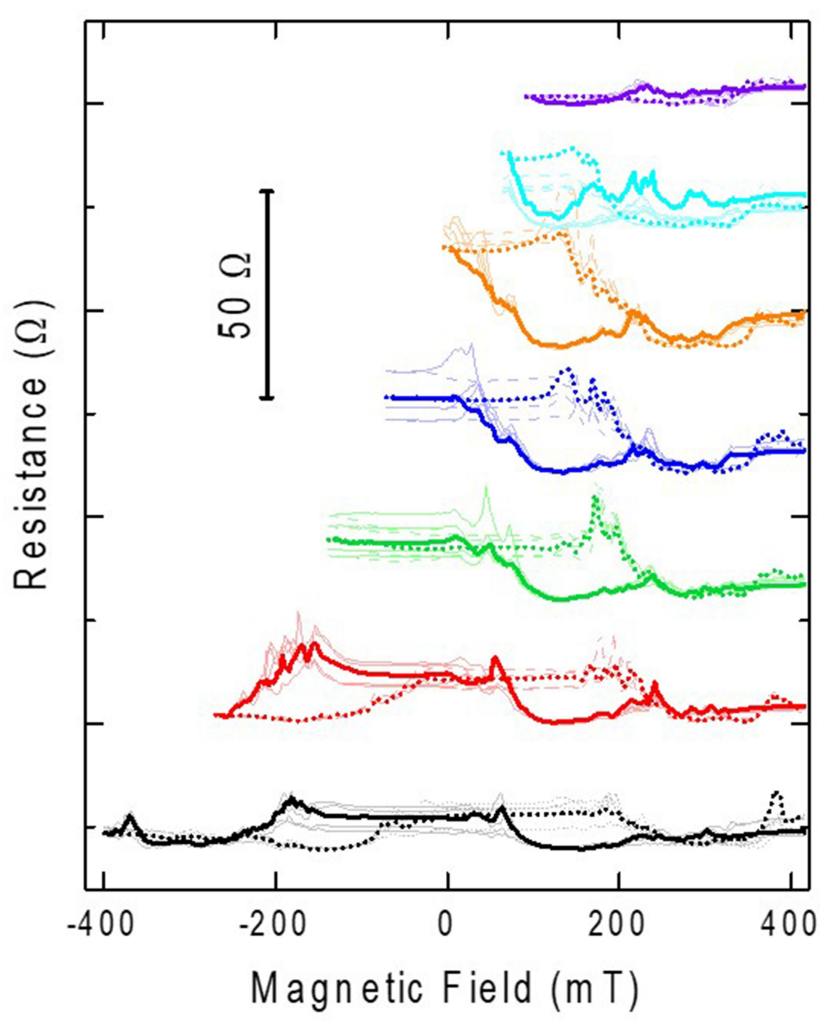

Figure 3 Major (black/gray) and minor (colored) magnetoresistance loops showing magnetic hysteresis of the tungsten point contact at $0.7 \mathrm{~mA}$ bias. Each measurement starts at a positive field just over $+400 \mathrm{mT}$. Solid (dotted/dashed) curves depict magnetic field down-sweeps (up-sweeps). Minor loops with different turning points were shifted vertically for clarity. Turning point fields are $-400,-260,-130,-60,0$, 70 , and $100 \mathrm{mT}$. Lighter colored curves indicate down-sweep/up-sweep repetitions of the same measurement while thicker traces highlight representative sweeps. were shifted vertically for clarity; turning-point fields are $-400 \mathrm{mT}$ (black/grey), $-260 \mathrm{mT}$ (red), $-130 \mathrm{mT}$ (green), $-60 \mathrm{mT}$ (blue), $0 \mathrm{mT}$ (orange), $70 \mathrm{mT}$ (cyan), and $100 \mathrm{mT}$ (purple). Lighter colored curves indicate down-sweep/upsweep repetitions of the same measurement while thicker traces highlight representative sweeps. The turning point of the first minor loop (red) coincides roughly with the resistance crossover between high and low resistance states in the major loop, effectively capturing the $50 \%$ MR behavior at the smallest negative value magnetic field. The second minor loop (green) with its turning point of $-130 \mathrm{mT}$ does not access the low resistance state associated with high negative magnetic fields. Furthermore, the third (blue) and fourth (orange) minor loops all show a similar behavior of remaining in the high resistance state near their turning points. The fifth minor loop (cyan) with its turning point at $70 \mathrm{mT}$ shows a hysteretic opening with slightly less area than the previous three minor loops, while the last minor loop (purple) fails to produce an appreciable hysteretic opening and remains in the low resistance state. The observed behavior is fully consistent with the switching between the parallel $\left(R_{L}\right)$ and antiparallel $\left(R_{H}\right)$ states in a spin valve or magnetic tunneling junction., ${ }^{6,7}$

Figure 4A shows an example of point-contact magnetoresistance with an MR ratio of $>10,000 \%$. Here red and black curves show magnetic field down- and up-sweeps, respectively, for a high-resistance tungsten point contact under a relatively low applied bias of $0.1 \mathrm{~mA}$. Compared with the contact from Figure 2 the principal modification is the resistance of the high resistance state $\left(R_{H}\right)$, while the low resistance state $\left(\mathrm{R}_{\mathrm{L}}\right)$ and the general form of the MR curve are mostly retained. At high fields, where all layers in the multilayer stack are presumably saturated, the resistance $R_{L}$ is measured at $20 \Omega$, while at modest fields there are large variations in resistance hovering between $2 \mathrm{k} \Omega$ and $6 \mathrm{k} \Omega$. We observed a strong dependence of the MR on the bias current applied to the contact. Lighter colored curves in Figure 4A show MR of the same device under a different (higher) bias current of $1.5 \mathrm{~mA}$ that results in a $1500 \%$ MR ratio. Figure $4 \mathrm{~B}$ shows that the MR ratio decreases with increasing bias independent of the bias polarity. We note that the main change in the magnitude of MR originates in the bias dependence of $R_{H}$ while $R_{L}$ remains relatively intact. Such a bias dependence of the contact magnetoresistance is very similar to the one well established in magnetic tunneling junctions (MTJs), ${ }^{6,8,9}$ where this behavior is usually associated with magnon- 

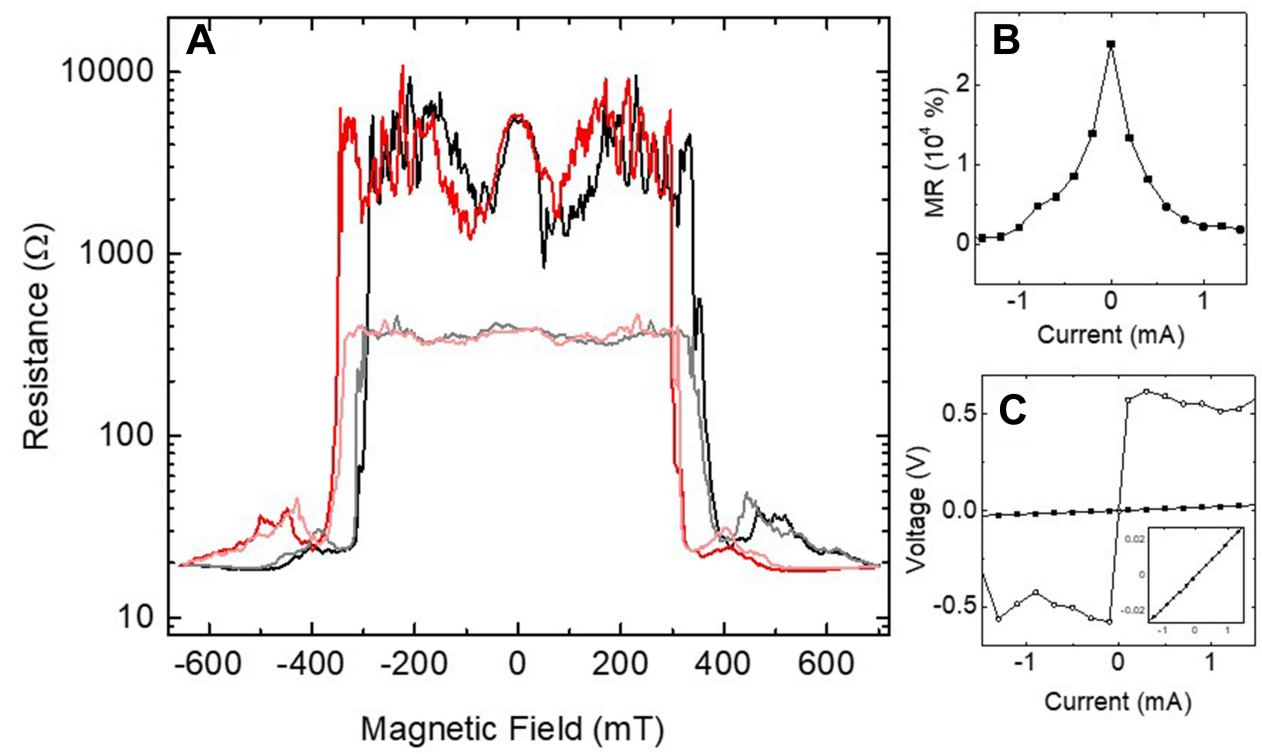

Figure 4 (A) Magnetoresistance (MR) trace of tungsten point-contact device displaying vast variation in resistance from $20 \Omega$ to $4 \mathrm{k} \Omega$ corresponding to $>10,000 \%$ MR. Red (black) curve depicts magnetic field down-sweep (up-sweep) recorded at low applied bias of $0.1 \mathrm{~mA}$. Lighter colored curves show MR of the same device under a different (higher) bias current of I.5 mA (I500\% MR ratio). (B) The bias dependence of MR ratio. (C) The current-voltage curves at $0 \mathrm{mT}$ (open circles) and $700 \mathrm{mT}$ (solid squares). The inset shows a zoom into the $700 \mathrm{mT}$ curve.

assisted and intrinsic band-structure mechanisms of bias dependence as well as phonon- and impurity-assisted contributions. Figure 4C shows the current-voltage curves at $0 \mathrm{mT}$ (open circles) and $700 \mathrm{mT}$ (solid squares); the inset of Figure 4C zooms into the $700 \mathrm{mT}$ curve. Here the lowfield $(0 \mathrm{mT})$ curve indicates an increase in the contact $\mathrm{R}_{\mathrm{H}}$ resistance with decreasing bias, which is consistent with the MTJ behavior. In contrast, the high-field (700 mT) curve displays a metallic-like behavior that correlates with an almost bias-independent $\mathrm{R}_{\mathrm{L}}$.

A point contact between two metallic electrodes at room temperature usually assumes a diffusive metallic conduction, ${ }^{7}$ as ballistic resistance can be ruled out due to the short mean free path of electrons at room temperature. ${ }^{10,11}$ However, neither diffusive no ballistic regime can explain the very high resistance $\mathrm{R}_{\mathrm{H}}$ (almost $10 \mathrm{k} \Omega$ ) of our point contact in Figure 4; such a high resistance value is usually associated with tunneling contacts. We speculate that the large resistance changes demonstrated in Figure 4 may be caused by tunneling effects, which we would expect to be relevant in our composite sample material. The Co-Pt-Cr granular media under study has a hexagonal columnar structure that is perpendicular to the plane and would presumably support high current densities in the perpendicular direction. The perpendicularly magnetized columns in adjacent magnetic layers of our multilayered samples would serve as channels for these perpendicular currents. However, an antiparallel orientation of magnetizations in the adjacent layers (layers with different coercivities separated by spacer layers/oxide barriers) may hinder the perpendicular current flow, perhaps bringing it to a point on par with the small in-plane currents between channels, ie, across the oxide segregation barriers. If perpendicular currents and in-plane currents have similar path resistances the total perpendicular current will be funneled to the relatively few magnetic domain interfaces that exhibit aligned orientations. This process, active under low (non-saturating) applied fields, would effectively create a network of tunneling junctions composed of both intralayer and interlayer tunneling junctions leading to possibly very large resistance $\left(R_{H}\right)$. In contrast, a magnetic saturation at higher fields would promote a parallel orientation of magnetizations in the adjacent layers and opening a low-resistance $\left(\mathrm{R}_{\mathrm{L}}\right)$ perpendicular channel. Observing such behavior would require a probe with a similar contact area as the magnetic grain size, namely, 7-9 $\mathrm{nm}$ diameter since probes with larger areas would average over many grains overlooking the nanoscale behavior. Note that recent measurements of tunneling magnetoresistance (TMR) from larger areas of granular magnetic media (with device geometry of $\sim 100 \mathrm{~nm}$ or larger) have reported a small TMR ratio of $0.23 \%$, which is possibly attributed to inter-granular averaging effects. ${ }^{12}$ Achieving a nanoscale probe area has motivated the introduction of a tungsten point-contact tip instead of copper as discussed above. Tungsten tip is 
expected to deform less when contacting the sample surface due to its hardness and will be more mechanically stable so as to minimize the impact of sample's magnetostrictive effect. $^{13}$ Tungsten point contacts would therefore possess a smaller contact area more suitable for investigating the nanoscale behavior of the granular composite media and associated large resistance changes caused by the proposed tunneling junction network.

\section{Conclusions}

In conclusion, we investigated the bulk and local transport properties of CoPtCr granular composite media films. The bulk shows essentially no magnetoresistance. In contrast, local (point-contact) measurements revealed giantmagnetoresistance-like changes in resistance with $>10,000 \%$ ratios. The observed magnetoresistive effect is tentatively attributed to a tunneling magnetoresistance between $\mathrm{CoPtCr}$ grains with different coercivities. The observed effects support the feasibility of a novel concept of magnetic memory based on granular composites where reading and writing operations would be achieved by magnetoresistive transport means.

\section{Acknowledgments}

This work was supported in part by the Sponsored Research Agreement No. UTA18-000691 between the University of Texas at Austin and Seagate Technology.

\section{Disclosure}

The authors report no conflicts of interest in conducting this work.

\section{References}

1. Mee CD, Daniel ED. Magnetic Recording Technology. 2nd ed. McGraw-Hill Professional; 1996.

2. Weller D, Parker G, Mosendz O, et al. A HAMR media technology roadmap to an areal density of $4 \mathrm{~Tb} / \mathrm{in}^{2}$. IEEE Trans Magn. 2013;50 (1): $1-8$.

3. Tsoi M, Jansen AGM, Bass J, et al. Excitation of a magnetic multilayer by an electric current. Phys Rev Lett. 1998;80:4281-4284. doi:10.1103/PhysRevLett.80.4281

4. Bian B, Wang S, Xu W, et al., inventors; Seagate Technology LLC, assignee. Magnetic recording media with enhanced writability and thermal stability. United States patent US 8685547. 2014 Apr 1.

5. Lu B, Weller D, Ju G, et al. Development of Co-alloys for perpendicular magnetic recording media. IEEE Trans Magn. 2003;39:1908. doi:10.1109/TMAG.2003.813779

6. Tsymbal EY, Zutic I, eds. Handbook of Spin Transport and Magnetism. 2nd ed. CRC press; 2019:313

7. Tsoi M, Jansen AGM, Bass J. Search for point-contact giant magnetoresistance in Co/Cu multilayers. J Appl Phys. 1997;81:5530-5532. doi:10.1063/1.364944

8. Julliere M. Tunneling between ferromagnetic films. Phys Lett. 1975;54A(3):225-226. doi:10.1016/0375-9601(75)90174-7

9. Moodera JS, Kinder LR, Wong TM, Meservey R. Large magnetoresistance at room temperature in ferromagnetic thin film tunnel junctions. Phys Rev Lett. 1995;74(16):3273-3276. doi:10.1103/ PhysRevLett.74.3273

10. Takayanagi K, Kondo Y, Ohnishi H. Suspended gold nanowires: ballistic transport of electrons. JSAP Int. 2001;3:9.

11. Datta S. Electronic Transport in Mesoscopic Systems. New York: Cambridge University Press; 1997:57-111.

12. Dieny B, Chshiev M, Charles B, et al. Impact of intergrain spin transfer torques due to huge thermal gradients on the performance of heat assisted magnetic recording. Presented at: IEEE International Magnetic Conference (INTERMAG). April 23-27;2018; Singapore, Singapore.

13. Egelhoff WF Jr., Gan L, Ettedgui H, et al. Artifacts in ballistic magnetoresistance measurements. J Appl Phys. 2004;95:7554. doi:10.1063/1.1688533
Nanotechnology, Science and Applications

\section{Publish your work in this journal}

Nanotechnology, Science and Applications is an international, peerreviewed, open access journal that focuses on the science of nanotechnology in a wide range of industrial and academic applications. It is characterized by the rapid reporting across all sectors, including engineering, optics, bio-medicine, cosmetics, textiles, resource sustainability and science. Applied research into nano-materials, particles,

\section{Dovepress}

nano-structures and fabrication, diagnostics and analytics, drug delivery and toxicology constitute the primary direction of the journal. The manuscript management system is completely online and includes a very quick and fair peer-review system, which is all easy to use. Visit http://www.dovepress.com/testimonials.php to read real quotes from published authors. 